\title{
DETECTION OF AN OPTICAL HALO SURROUNDING THE SPIRAL GALAXY NGC 4565
}

Dennis J. Hegyi

A halo surrounding the edge-on Sb giant spiral galaxy NGC 4565 has been detected in the spectral band 3800-8600 A using a new photometric instrument, an annular scanning photometer. The halo is brightest close to the galactic nucleus and decreases in brightness until it reaches a level of 1 part in 1000 of the sky at a galactic radius of 6.1 arc minutes or $43 \mathrm{kpc}$ from the galactic center. Because the scan path of the ASP is circular, this point corresponds to a distance of $34 \mathrm{kpc}$ from the galactic plane. For comparison, the Holmberg radius of NGC 4565 is equal to 7 arc minutes or $50 \mathrm{kpc}$. Preliminary V-I photometric data indicate that the halo becomes redder with increasing galactic radius, exceeding $\mathrm{V}-\mathrm{I}=1.9$. Based on a variety of possible origins for the light, it is concluded that the light is due to stars.

The rotation curve of NGC 4565 has been observed to be flat out to $100 \mathrm{kpc}$ by Krumm and Salpeter (1977) from which the authors deduce a galactic mass exceeding $10^{12} \mathrm{M}_{\odot}$. The combination of the optical and radio data suggest that the mass necessary to explain the flat rotation curve is contained in a massive stellar halo. Other authors (Einasto et a1. 1974, Ostriker, Peebles and Yahil 1974) have pieced together a number of dynamical arguments which indicate that spherical halos may make the dominant contribution to the cosmological mass density.

\section{REFERENCES}

Einasto, J., Kaasik, A. and Saar, E., 1974. Nature, 250, 309. Krumm, N. and Salpeter, E.E., 1977. Astron. and Astrophys., 56, 465. Ostriker, J.P., Peebles, P.J.E. and Yahi1, A., 1974. Ap. J. (Letters) 193, L1.

\section{DISCUSSION}

Ginzburg: Is there a radio halo around NGC 4565.

Hegyi: The galaxy is a source of synchrotron radiation but the extrapolation of its total radio flux density to optical wavelengths results in an optical flux density only $10^{-3}$ of our results.

Ostriker: The results you show appear to agree with the final results H. Spinrad and I derived for the same galaxy (NGC 4565) by more or less standard photographic and photoelectric methods.

Hegyi: Your results indicate a much brighter halo than we find by perhaps as much as an order of magnitude. 
Huchra: What is the bolometric luminosity of the halo of NGC 4565 and how much does it contribute to the total light?

Hegyi: The halo does not contribute a significant fraction of the total light.

Tinsley: What is the luminosity of the halo and what is your estimate of its $\mathrm{M} / \mathrm{L}$ ratio?

Hegyi: All I can say is that the M/L value must be very large. It is difficult to quote a single value for the luminosity because our observations are made in a very broad wavelength band.

Ekers: It is correct that NGC 4565 does not possess a radio halo but it is also odd in that the disc emission is rather weak. I might also add that recent Westerbork HI observations disagree with the results of Krumm and Salpeter.

Zasov: Can you compare the luminosity distribution along the major and minor axis of the galaxy?

Hegyi: The particular scan paths which we have chosen are relatively insensitive to the eccentricity of halo brightness distribution. The only conclusion that may be drawn is that our observations are consistent with both a spherical halo and a slightly eccentric one.

Silk: What is the central surface brightness extrapolated from your halo fit and how does it compare with that for elliptical galaxies?

Hegyi: An extrapolation of the de Vaucouleurs $\mathrm{r}^{\frac{1}{4}}$ law to the galactic centre would yield a higher surface brightness than that of most elliptical galaxies.

Peebles: Have you tried fitting your data to a Hubble law?

Hegyi: We did not try very hard but my present impression is that the de Vaucouleurs $\mathrm{r}^{\frac{1}{4}}$ law gives a better fit.

\section{THE SCATTER IN MASS-TO-LUMINOSITY RATIOS}

\section{Jaan Einasto}

Mass-to-luminosity ratios of systems of galaxies as derived by different authors have a large scatter from $\sim 10$ to $\sim 200$. The observational data used by different authors differ only slightly, so the differences should lie in the treatment of the data. We have found that in most cases $<M / L>$ differences can be explained in the following ways.

1. The relative motion of companion galaxies, located at a mean distance $R$ from the main galaxy, gives the inner mass $M(R)$ of the 